\title{
Prevalence of Candida dubliniensis among Oral Candida Isolates in Patients Attending the Kuwait University Dental Clinic
}

\author{
Arjuna N.B. Ellepola ${ }^{\mathrm{a}}$ Zia U. Khan ${ }^{c}$ Bobby Joseph ${ }^{\mathrm{b}}$ Rachel Chandy ${ }^{\mathrm{c}}$ \\ Leeba Philip ${ }^{a}$
}

Departments of ${ }^{a}$ Bioclinical and ${ }^{b}$ Diagnostic Sciences, Faculty of Dentistry, and ${ }^{c}$ Department of Microbiology, Faculty of Medicine, Health Sciences Centre, Kuwait University, Jabriya, Kuwait

\section{Key Words}

Prevalence $\cdot$ Candida $\cdot$ Oral flora

\begin{abstract}
Objectives: The aim of this study was to determine the oral candidal carriage of patients seeking dental treatment at the Kuwait University Dental Clinic and to ascertain the Candida species composition among them. Methods: 370 oral rinse samples were collected from patients. The germ tube test, CHROMagar Candida medium and VITEK 2 yeast identification system were used for species identification. C. dubliniensis isolates were confirmed by the production of rough colonies with hyphal fringes and chlamydospores on simplified sunflower seed agar. Results: Of the 370 samples investigated, 160 (43.24\%) showed Candida in culture. The isolation of Candida was significantly higher in individuals who were smokers or were under medication for either diabetics or asthma [99 (62\%)] compared to healthy individuals [61 (38\%)]. Of the 210 samples which did not yield Candida, 131 $(62.38 \%)$ were healthy and 79 (37.62\%) were associated with smoking or with usage of drugs for aforementioned conditions. Species isolated were C. albicans [102 (63.7\%)], C. dubliniensis [23 (14.3\%)], C. krusei [13 (8.1\%)], C. tropicalis [12 (7.5\%)] and C. glabrata [10 (6.2\%)]. Conclusions: Candida species
\end{abstract}

were more prevalent in patients having predisposing factors implicated in oral candidosis, such as in smokers, diabetic patients and asthmatic patients using inhalation steroids. C. albicans was the most prevalent species isolated, followed by $C$. dubliniensis.

Copyright $\odot 2011$ S. Karger AG, Basel

\section{Introduction}

Due to the increasing number of immunocompromised hosts, infections caused by opportunistic pathogens have become a serious problem worldwide. Species of Candida are among the most important opportunistic pathogens, which constitute part of the normal oral microbiota. Since species of Candida differ in virulence properties and susceptibility to antifungal drugs, understanding the human commensal flora will have a significant impact on designing treatment and prevention strategies against yeast infection. Although opportunistic yeast infections are considered a global problem and there is global interest in these causal agents, we know relatively little of the global distribution of Candida pathogens. Therefore, it is worth investigating the distribution of these pathogenic yeasts in different geographical locations.

\section{KARGER}

Fax +4161306 1234

E-Mail karger@karger.ch

www.karger.com
C 2011 S. Karger AG, Basel

$1011-7571 / 11 / 0203-0271 \$ 38.00 / 0$

Accessible online at:

www.karger.com/mpp
Dr. Arjuna N.B. Ellepola, BDS, PhD, FIBiol

Department of Bioclinical Sciences, Faculty of Dentistry, Health Sciences Centre Kuwait University, PO Box 24923

Safat 13110 (Kuwait)

Tel. +965 2498 6714, Fax +965 2532 6049, E-Mail arjuna@ hsc.edu.kw 
Most data on the distribution of pathogenic yeast species have come from patient populations in Europe and North America. These studies have reported yeast carriage rates of about $20-40 \%$ in typical population samples $[1,2]$. In these studies, C. albicans has been found to be the dominant species, accounting for up to about $90 \%$ of all isolated yeasts. In samples obtained from patients in developing countries such as those in Africa, the yeast carriage rates were similar to or higher than those reported in developed countries [3]. Interestingly, the diversity of yeast species was typically higher in developing countries, and the frequency of $C$. albicans was usually much lower than frequencies found in developed countries [3]. Several other surveys showed that the distribution of opportunistic yeast pathogens from healthy hosts in North America was similar to that in patient populations in North America and most other Western countries regarding both the yeast carriage rate and the yeast species distribution, with C. albicans as the dominant species [2]. In addition, different groups of healthy hosts in Hong Kong have yeast carriage rates and yeast species compositions highly similar to those in North America and Europe [4]. In contrast, a comparable survey of healthy hosts in several regions of mainland China identified a significantly higher rate of yeast carriage (66.9\%) than those reported in other regions [5]. Furthermore, C. albicans was ranked only fourth most prevalent, accounting for only $9.4 \%$ of all isolated yeast strains [5].

C. dubliniensis is an emerging pathogen capable of causing oropharyngeal, vaginal and systemic infections [6]. Though similar to C. albicans in several phenotypic characteristics, it differs from it with respect to epidemiology, certain virulent attributes and the ability to rapidly develop resistance to fluconazole [6]. There have been no previous studies on oral yeast carriage in the Middle Eastern population in general, and in the Kuwaiti population in particular. Furthermore, the prevalence of C. dubliniensis isolates among oral Candida isolates in this segment of the population is unknown. As such a study would add further knowledge to the understanding of the global distribution of this opportunistic species, the main aim of this study was to determine the oral candidal carriage of patients seeking dental treatment at the Kuwait University Dental Clinic (KUDC), and to ascertain the Candida species composition among the yeast carriers. The other aim was to determine the antifungal susceptibility of these isolates to antifungal drugs such as amphotericin $\mathrm{B}$, fluconazole, ketoconazole and caspofungin.

\section{Subjects and Methods}

\section{Study Group}

A total of 370 patients attending the KUDC for dental treatment were enrolled in the study. The KUDC is affiliated to the Faculty of Dentistry and situated in the Jabriya Kuwait University Campus and functions as an outpatient clinic. The KUDC provides a full range of dental treatment for those who have dental treatment needs that correspond to the teaching needs of dental students. Hence, in general, the majority of the patients attending the clinic do not belong to the category of having an increased risk for higher carriage of oral Candida. The ethical clearance for this study was granted by the Health Sciences Center Ethics Clearance Committee, Faculty of Medicine, Kuwait University, and the study was undertaken with the informed consent of each patient. Of the total of 370 patients, 176 were males (16-67 years) and 194 were females (17-71 years). All patients came for a routine dental checkup at the time of attending the KUDC for dental treatment. There were 178 patients (107 males and 71 females) who were heavy smokers, while others were under treatment for diabetes mellitus and bronchial asthma. The diabetic patients were under oral hypoglycemic drugs and the asthmatic patients under steroid inhalation therapy, and they were otherwise healthy at the time of attending the KUDC. The patients who smoked more than 25 cigarettes per day were categorized as heavy smokers. For the purpose of description, this group is identified as 'compromised patients' in the text. The remaining 192 patients (69 males and 123 females) were not associated with either smoking or medication. None of the 370 patients had clinically proven oral candidosis.

\section{Isolation and Identification}

The standard oral rinse technique was used for the isolation of Candida species. All yeast isolates were tested for germ tube formation. Thereafter, a presumptive identification of the Candida isolates was performed on the basis of the characteristic colony color on CHROMagar Candida medium (Becton, Dickinson and Company, Sparks, Md., USA). All the isolates were further identified based on their carbohydrate assimilation pattern by the VITEK 2 yeast identification system (bioMérieux, Craponne, France). The identity of C. dubliniensis was confirmed by the production of rough colonies with hyphal fringes and chlamydospores on simplified sunflower seed agar [7], which has shown to accurately identify $C$. dubliniensis when compared with molecular identification methods using seminested PCR amplification of the internal transcribed spacer-2 region of rDNA followed by direct DNA sequencing of the internal transcribed spacer region of rDNA as described previously [8].

\section{Antifungal Susceptibility}

Antifungal susceptibility of the yeast isolates was determined against amphotericin B, fluconazole, ketoconazole and caspofungin by E test performed according to the manufacturer's recommendations (AB BIODISK, Solna, Sweden). Each test isolate was freshly subcultured. Five isolated colonies were uniformly suspended in sterile saline, and turbidity was adjusted to $0.5 \mathrm{McFar}-$ land standard. This inoculum was swabbed onto the agar plates (150 $\mathrm{mm}$ diameter) and allowed to dry for 10-15 min before the E test strips were applied. RPMI 1640 agar supplemented with $2 \%$ glucose and buffered with MOPS $(0.165 \mathrm{M}$; pH 7.0) was used for susceptibility testing according to the method recommended by 
Table 1. Variance in the distribution of patients $(n=160)$ who yielded Candida in culture (n)

\begin{tabular}{lrrl}
\hline & \multicolumn{2}{l}{ Gender distribution } & $\begin{array}{l}\text { Number } \\
\text { of patients }\end{array}$ \\
\cline { 2 - 3 } & $\begin{array}{l}\text { males } \\
(\mathrm{n}=67 ; 42 \%)\end{array}$ & $\begin{array}{l}\text { females } \\
(\mathrm{n}=93 ; 58 \%)\end{array}$ \\
\hline Healthy & 14 & 47 & 61 \\
Smoking & 34 & 0 & 34 \\
Medication for diabetes & 2 & 29 & 31 \\
Medication for asthma & 16 & 11 & 27 \\
Other & 1 & 6 & 7 \\
\hline Total & 67 & 93 & $160(100 \%)$ \\
\hline
\end{tabular}

the Clinical and Laboratory Standards Institute (formerly National Committee for Clinical Laboratory Standards; M27-A2). The plates were incubated at $35^{\circ} \mathrm{C}$, and minimum inhibitory concentrations (MIC) were read after $24-48 \mathrm{~h}$ of incubation. The point where inhibition ellipses intercepted the scale on the antifungal strip was taken as the MIC for each test isolate: complete inhibition (100\%) of growth for amphotericin B and caspofungin, marked decrease in growth intensity $(80 \%)$ for fluconazole and ketoconazole. Reference strains of C. albicans, ATCC 90028, and C. parapsilosis, ATCC 22019, were used for quality control of the susceptibility testing. Interpretive susceptibility breakpoints for fluconazole were those recommended by Clinical and Laboratory Standards Institute document M27-A2. Due to the lack of defined susceptibility breakpoints for amphotericin B and ketoconazole, an isolate was considered susceptible with an MIC breakpoint of $\leq 1.0 \mu \mathrm{g} / \mathrm{ml}$ for amphotericin B and $0.125 \mu \mathrm{g} / \mathrm{ml}$ for ketoconazole. For caspofungin, an isolates with MIC of $\leq 2 \mu \mathrm{g} / \mathrm{ml}$ were considered as susceptible.

\section{Statistical Analysis}

To compare the potential differences in yeast carriage rates among groups of hosts, the $\chi^{2}$ contingency table test was used. The differences among the compromised and healthy groups were analyzed, and a $\mathrm{p}<0.05$ was considered significant.

\section{Results}

Prevalence and Distribution of Oral Candida Species

Of the 350 patients investigated, 160 (43.24\%) yielded Candida in culture; 93 (58\%) were females and 67 (42\%) males. The isolation rate of Candida was higher in individuals who were compromised in addition to the dental complaint [99 (62\%); 46 females and 53 males] compared to healthy individuals ( $\mathrm{p}<0.01$ ). Among the individuals who had only the dental complaint, the isolation rate was only $38 \%$ ( $n=61 ; 47$ females and 14 males). The distribution of the patients who yielded Candida in culture is giv-
Table 2. Distribution and percentage of different oral Candida species in 160 patients that yielded Candida in culture

\begin{tabular}{lccc}
\hline & Distribution $A^{1}$ & Distribution $B^{2}$ & Total species \\
\hline C. albicans & 73 & 29 & $102(63.7 \%)$ \\
C. dubliniensis & 11 & 12 & $23(14.3 \%)$ \\
C. krusei & 7 & 6 & $13(8.1 \%)$ \\
C. tropicalis & 6 & 6 & $12(7.5 \%)$ \\
C. glabrata & 2 & 8 & $10(6.2 \%)$ \\
\hline Total & 99 & 61 & $160(100 \%)$
\end{tabular}

${ }^{1}$ Distribution in patients with dental complaint associated with either heavy smoking or usage of medication.

${ }^{2}$ Distribution in healthy patients with dental complaint only.

en in table 1. The distribution and percentage of different oral Candida species in these 160 patients that yielded Candida in culture are given in table 2. Accordingly, the species isolated were C. albicans [102 (63.7\%)], C. dubliniensis [23 (14.3\%)], C. krusei [13 (8.1\%)], C. tropicalis [12 (7.5\%)] and C. glabrata [10 (6.2\%)]. The isolation of C. albicans in the compromised group was significantly higher $(n=73)$ than in the group which only had the dental complaint $(\mathrm{n}=29)$. Of the 180 samples which did not yield any Candida, 131 (62.38\%) were healthy apart from the dental complaint, and only 79 (37.62\%) were compromised patients in addition to the dental complaint. This difference too was statistically significant $(p<0.01)$.

\section{Antifungal Susceptibility}

The data on antifungal susceptibility as determined by the E test are presented in table 3. All Candida isolates tested were susceptible to amphotericin B and caspofungin. All C. albicans, C. dubliniensis and C. tropicalis isolates were also susceptible to fluconazole and ketoconazole. C. glabrata isolates showed reduced susceptibility to fluconazole and some were determined as resistant to ketoconazole. However, all C. krusei were determined as resistant to fluconazole and ketoconazole.

\section{Discussion}

The present study to our knowledge is the first study to survey the prevalence of oral Candida species in a Middle Eastern country. Of the 370 patients investigated, 43.24\% yielded Candida in culture. Previous studies in Europe and North America have reported yeast carriage rates of about $20-40 \%[1,2]$. A slightly higher oral candi- 
Table 3. Antifungal susceptibility of different oral Candida species by E test $(\mu \mathrm{g} / \mathrm{ml})$

\begin{tabular}{lllccc}
\hline & C. albicans & C. dubliniensis & C. krusei & C. tropicalis & C. glabrata \\
\hline Amphotericin B & $0.004-0.19$ & $0.002-0.125$ & $0.008-0.25$ & $0.004-0.38$ & $0.032-0.25$ \\
Ketoconazole & $0.004-0.032$ & $0.002-0.012$ & $0.19-0.75$ & $0.012-0.19$ & $0.008-0.38$ \\
Fluconazole & $0.047-0.125$ & $0.016-0.38$ & $32-64$ & $0.38-1.5$ & $2-16$ \\
Caspofungin & $0.004-0.125$ & $0.003-0.19$ & $0.004-0.5$ & $0.004-0.38$ & $0.032-0.19$ \\
\hline
\end{tabular}

Values denote MIC ranges.

dal carriage rate of $53 \%$ has been reported in a study conducted in South Africa [9]. The observed prevalence of candidal carriage of $43.24 \%$ falls within the range of 20 $60 \%$ reported by other researchers [10]. The results of the present study also showed a higher prevalence of Candida in females than in males, similar to other studies [11]. The isolation rate of Candida was significantly higher in individuals who were associated with smoking or were receiving medication for diabetes and asthma in addition to the dental complaint (62\%) compared to individuals who only had the dental complaint (38\%). Hence, the current finding on the prevalence of Candida in the oral cavity further adds credence to the fact that Candida is an opportunistic pathogen.

On the distribution of different Candida species among the patients who yielded Candida in culture, our analysis identified that $C$. albicans was the dominant yeast species in our sample, accounting for $63.7 \%$ of all Candida isolates. Previous studies in North America and Europe have also reported C. albicans to be the dominant species isolated, accounting to up to $90 \%$ of all isolates tested $[1,2]$. Others have revealed that though the most dominant, the frequency of $C$. albicans could be lower than frequencies found in North America and Europe [3]. Moreover, C. albicans was ranked fourth most prevalent, accounting for only $9.4 \%$ of all isolates tested, in a study conducted in mainland China [5]. Hence, our finding falls within the range of previous findings on the prevalence of $C$. albicans species $[1-3,5]$.

C. glabrata and C. tropicalis are considered as the commonest non-albicans oral Candida species isolated after C. albicans [12]. In contrast, in our sample, among the non-albicans Candida species isolated, C. dubliniensis was the most dominant species, accounting for $14.3 \%$ of all Candida species tested. Conversely, in studies on oral carriage of Candida species in patient populations involving both healthy and compromised, but not HIV-positive subjects, $C$. dubliniensis was not isolated at all among all Candida species tested [13-17]. Furthermore, in another study on carriage of Candida species in the oral cavities of HIV-infected patients and healthy individuals in South India, C. dubliniensis was not isolated among all Candida species tested [18]. However, in a study on oral yeast carriage in HIV-infected populations in Argentina, the isolation rate of $C$. dubliniensis was $20.2 \%$ in HIVpositive patients, whereas it was $2.1 \%$ in HIV-negative individuals [19]. The isolation rate of this pathogen in HIVpositive patients in a study conducted in India was $16.3 \%$ of all Candida species isolated [20]. C. dubliniensis is now well recognized as an opportunistic pathogen associated with recurrent oral candidiasis in HIV infection, and this could be the reason for the findings of the later two studies. In a study with diabetic patients conducted in Ireland, the isolation rate of $C$. dubliniensis was also higher, with $18.2 \%$ of the Candida species isolated being C. dubliniensis [21]. In contrast, in another study with diabetic patients conducted in Turkey, none of the Candida isolates recovered were C. dubliniensis [22]. However, compared to all the aforementioned studies, in our study none of the patients were HIV-positive but were healthy, even though some patients were smokers and some were under medication for diabetes and asthma. Hence, taken together, our finding suggests a high prevalence of oral carriage of C. dubliniensis isolates compared to the majority of previous studies. Although the exact reasons for such differences in diversity remain unknown, several reasons could be proposed for this high prevalence rate as seen in our study. Firstly, there may be a racial preference of C. dubliniensis isolates for the tested population. In fact, a study by McCullough et al. [23] reported that genotypic differences among C. dubliniensis may be related to ethnic or racial differences between Jews, Arabs and Druse, despite the fact that they inhabit the same geographic area. A racial preference of $C$. dubliniensis has also been suggested in another study from South Africa, where $16 \%$ of oral cavities of healthy white individuals were colonized with 
the species, whereas the isolation rate among healthy blacks was $0 \%$ [24]. Secondly, C. dubliniensis is closely related to C. albicans in evolutionary terms, sharing the latter's properties of commensalism and opportunistic infection with genomic similarities between these two species, with the vast majority of genes being approximately $90 \%$ homologous. Given this similarity, it is not surprising that previous studies may have misidentified C. dubliniensis as C. albicans due to the unavailability of reliable methods for accurately differentiating these two species [25]. However, analysis of data from a larger number of the population in Kuwait is important before arriving at these conclusions.

All Candida isolates tested were susceptible to amphotericin B and caspofungin. Furthermore, all C. albicans, C. dubliniensis and C. tropicalis isolates were also susceptible to fluconazole and ketoconazole. However, C. glabrata isolates showed reduced susceptibility to fluconazole and some of these isolates were also resistant to ketoconazole. All C. krusei isolates were resistant to both ketoconazole and fluconazole. Candida species differ in their susceptibility to antifungal agents. For instance, $C$. krusei is often innately azole resistant, C. glabrata has been reported to acquire resistance in vitro and in vivo, and $C$. dubliniensis isolates have been observed to rapidly develop resistance to fluconazole [26, 27]. In our study, too, all C. krusei isolates were resistant to both azoles (fluconazole and ketoconazole). C. glabrata showed reduced susceptibility to fluconazole and some of these isolates were also resistant to ketoconazole. Though C. dubliniensis isolates have been observed to rapidly develop resistance to fluconazole, all isolates in our study were suscep- tible to this drug. Reduced susceptibility as well as frank resistance to drugs such as fluconazole and ketoconazole, as observed in our study, could be an issue of clinical importance in a scenario if these patients develop oral candidal lesions. Hence, antifungal susceptibility testing could be a valuable tool for predicting the efficacy of a given agent, and could help guide empiric therapy for high-risk patients with known predisposing factors for developing serious candidal infection (i.e. smokers and patients under medication as seen in our study). On the other hand, identification of the infecting species such as C. krusei or C. glabrata could also be highly predictive of the likely drug susceptibility to azoles and could be used as a guide to therapy.

\section{Conclusion}

C. albicans was the most prevalent species isolated from the oral cavity, followed by $C$. dubliniensis, in the population tested. Compared to other prevalence studies on oral candidal microbiota, a high prevalence of $C$. $d u b$ liniensis over other non-albicans species was seen in this study. Analysis of data from a larger number of the population in Kuwait is important before arriving at this conclusion.

\section{Acknowledgment}

This study was supported by Kuwait University research grant No. DB01/07.

\section{References}

1 Hazen KC: New and emerging yeast pathogens. Clin Microbiol Rev 1995;8:462-478.

- Kleinegger CL, Lockhart SR, Vargas K, Soll DR: Frequency, intensity, species, and strains of oral Candida vary as a function of host age. J Clin Microbiol 1996;34:2246-2254.

-3 Okungbowa FI, Isikhuemhen OS, Dede AP: The distribution frequency of Candida species in the genitourinary tract among symptomatic individuals in Nigerian cities. Rev Iberoam Micol 2003;20:60-63.

$\checkmark 4$ Sedgley CM, Samaranayake LP: The oral prevalence of aerobic and facultatively anaerobic Gram-negative rods and yeasts in Hong Kong Chinese. Arch Oral Biol 1994;39: 459-466.
5 Xu J, Mitchell TG: Geographical differences in human oral yeast flora. Clin Infect Dis 2003;36:221-224.

-6 Sullivan DJ, Moran GP, Pinjon E, Al-Mosaid A, Stokes C, Vaughan C, Coleman DC: Comparison of the epidemiology, drug resistance mechanisms, and virulence of Candida dubliniensis and Candida albicans. FEMS Yeast Res 2004;4:369-376.

-7 Khan ZU, Ahmad S, Mokaddas E, Chandy R: Simplified sunflower (Helianthus annuus) seed agar for differentiation of Candida dubliniensis from Candida albicans. Clin Microbiol Infect 2004;10:590-592.
8 Ahmad S, Mokaddas E, Al-Sweih N, Khan $\mathrm{ZU}$ : Phenotypic and molecular characterization of Candida dubliniensis isolates from clinical specimens in Kuwait. Med Princ Pract 2005;14:77-83.

-9 Masipa JN, Hauman CH, Raubenheimer EJ: Oral carriage of Candida species in patients visiting the Medunsa Dental Clinic. J Dent Assoc S Afr 1992;47:407-409.

10 Epstein JB, Truelove EL, Izutzu KT: Oral candidiasis: pathogenesis and host defense. Rev Infect Dis 1984;6:96-106.

11 Bastiaan RJ, Reade PC: The prevalence of Candida albicans in the mouths of tobacco smokers with and without oral mucous membrane keratoses. Oral Surg Oral Med Oral Pathol 1982;53:148-151. 
12 Zhu HW, McMillan AS, McGrath C, Li LS, Samaranayake LP: Oral carriage of yeasts and coliforms in stroke sufferers: a prospective longitudinal study. Oral Dis 2008; 14 : 60-66.

13 Wang H, Wang Y, Chen J, Zhan Z, Li Y, Xu J: Oral yeast flora and its ITS sequence diversity among a large cohort of medical students in Hainan, China. Mycopathologia 2007; 164:65-72.

14 Thaweboon S, Thaweboon B, Srithavaj T, Choonharuangdej S: Oral colonization of Candida species in patients receiving radiotherapy in the head and neck area. Quintessence Int 2008;39:52-57.

$\rightarrow 15$ Reichart PA, Samaranayake LP, Bendick C, Schmidt-Westhausen AM, Jayatilake JA: Prevalence of oral Candida species in leprosy patients from Cambodia and Thailand. J Oral Pathol Med 2007;36:342-346.

16 de Araújo Navas EA, Inocêncio AC, Almeida JD, Back-Brito GN, Mota AJ, Jorge AO, Querido SM, Balducci I, Koga-Ito CY: Oral distribution of Candida species and presence of oral lesions in Brazilian leprosy patients under multidrug therapy. J Oral Pathol Med 2009;38:764-767.
7 Pinto E, Ribeiro IC, Ferreira NJ, Fortes CE, Fonseca PA, Figueiral MH: Correlation between enzyme production, germ tube formation and susceptibility to fluconazole in Candida species isolated from patients with denture-related stomatitis and control individuals. J Oral Pathol Med 2008;37:587-592.

18 Girish Kumar CP, Menon T, Rajasekaran S, Sekar B, Prabu D: Carriage of Candida species in oral cavities of HIV infected patients in South India. Mycoses 2009;52:44-48.

19 Luque AG, Biasoli MS, Tosello ME, Binolfi A, Lupo S, Magaró HM: Oral yeast carriage in HIV-infected and non-infected populations in Rosario, Argentina. Mycoses 2009; 52:53-59.

20 Nadagir SD, Chunchanur SK, Halesh LH, Yasmeen K, Chandrasekhar MR, Patil BS: Significance of isolation and drug susceptibility testing of non-Candida albicans species causing oropharyngeal candidiasis in HIV patients. Southeast Asian J Trop Med Public Health 2008;39:492-495.

21 Willis AM, Coulter WA, Sullivan DJ, Coleman DC, Hayes JR, Bell PM, Lamey PJ: Isolation of C. dubliniensis from insulin-using diabetes mellitus patients. J Oral Pathol Med 2000;29:86-90.

22 Tekeli A, Dolapci I, Emral R, Cesur S: Candida carriage and Candida dubliniensis in oropharyngeal samples of type- 1 diabetes mellitus patients. Mycoses 2004;47:315-318.
23 McCullough MJ, Jorge JJ, Lejbkowicz F, Lefler E, Nassar F, Clemons KV, Stevens DA: Genotypic differences of Candida albicans and C. dubliniensis isolates related to ethnic/ racial differences within the same geographic area. Mycopathologia 2004;158:39-41.

24 Blignaut E, Pujol C, Joly S, Soll DR: Racial distribution of Candida dubliniensis colonization among South Africans. J Clin Microbiol 2003;41:1838-1842.

25 Ellepola AN, Hurst SF, Elie CM, Morrison CJ: Rapid and unequivocal differentiation of Candida dubliniensis from other Candida species using species-specific DNA probes: comparison with phenotypic identification methods. Oral Microbiol Immunol 2003;18: 379-388.

26 Moran GP, Sanglard D, Donnelly SM, Shanley DB, Sullivan DJ, Coleman DC: Identification and expression of multidrug transporters responsible for fluconazole resistance in Candida dubliniensis. Antimicrob Agents Chemother 1998:42:1819-1830.

27 Trick WE, Fridkin SK, Edwards JR, Hajjeh RA, Gaynes RP: Secular trend of hospitalacquired candidemia among intensive care unit patients in the United States during 1989-1999. Clin Infect Dis 2002;35:627-630. 